\title{
Neuroprotective Mechanisms of Calycosin Against Focal Cerebral Ischemia and Reperfusion Injury in Rats
}

\author{
Yong Wang ${ }^{a, b}$ Qianyao Ren ${ }^{a} \quad$ Xing Zhang ${ }^{a}$ Huiling Lu, ${ }^{a, c}$ Jian Chen ${ }^{a}$ \\ aKey Laboratory of Tumor Immunology and Microenvironmental Regulation, Guilin Medical University, \\ Guilin, Guangxi, bDepartment of Physiology, Guilin Medical University, Guilin, Guangxi, 'Department of \\ Pathology and Physiopathology, Guilin Medical University, Guilin, Guangxi, China
}

\section{Key Words}

Calycosin·Cerebral • ischemia and reperfusion • Autophagy • Apoptosis • inflammation

\begin{abstract}
Background/Aims: Emerging evidence suggests that autophagy plays important roles in the pathophysiological processes of cerebral ischemia and reperfusion injury. Calycosin, an isoflavone phytoestrogen, possesses neuroprotective effects in cerebral ischemia and reperfusion in rats. Here, we investigated the neuroprotective effects of calycosin against ischemia and reperfusion injury, as well as related probable mechanisms behind autophagy pathways. Methods: A cerebral ischemic and reperfusion injury model was established by middle cerebral artery occlusion in male Sprague-Dawley rats. Neurological scores, infarct volumes, and brain water content were assessed after $24 \mathrm{~h}$ reperfusion following $2 \mathrm{~h}$ ischemia. Additionally, the expression of the autophagy-related protein p62 and NBR1 (neighbor of BRCA1 gene 1), as well as BCl-2, and TNF- $\alpha$ in rat brain tissues was measured by RT-PCR, western blotting and immunohistochemical analyses. Results: The results showed that calycosin pretreatment for 14 days markedly decreased infarct volume and brain edema, and ameliorated neurological scores in rats with focal cerebral ischemia and reperfusion. It was observed that levels of p62, NBR1 and Bcl-2 were greatly decreased, and levels of TNF- $\alpha$ significantly increased after ischemia and reperfusion injury. However, calycosin administration dramatically upregulated the expression of p62, NBR1 and Bcl-2, and downregulated the level of TNF- $\alpha$. Conclusions: All data reveal that calycosin exerts a neuroprotective effect on cerebral ischemia and reperfusion injury, and the mechanisms maybe associated with its antiautophagic, anti-apoptotic and anti-inflammatory action.

\section{Introduction}

Cerebral ischemia and reperfusion (I/R) injury is a complex of pathophysiological events linked to deprivaition of oxygen and energy from blood during the period of ischemia,

Y. Wang and Q. Ren contributed equally to this work.

Huiling Lu

and Jian Chen

KARGER
Key Laboratory of Tumor Immunology and Microenvironmental Regulation

Guilin Medical University, Guilin, Guangxi (China)

Tel.+86-773-5895800, E-Mail luhuiling7812@126.com, chenjian@glmc.edu.cn 


\section{Cellular Physiology Cell Physiol Biochem 2018;45:537-546 \begin{tabular}{ll|l} 
DOI: 10.1159/000487031 & $\begin{array}{l}\text { O 2018 The Author(s). Published by S. Karger AG, Basel } \\
\text { www.karger.com/cpb }\end{array}$
\end{tabular}}

Wang et al.: Neuroprotective Mechanisms of Calycosin

eventually resulting in neuronal destruction [1,2]. Pathogenic mechanisms following cerebral ischemia and reperfusion are associated with energy failure, elevation of the intracellular $\mathrm{Ca}^{2+}$ level, release of excitatory neurotransmitters, oxidative stress, inflammation, apoptosis, etc. [3]. Recent data indicate that autophagy is activated in cerebral ischemia and performs a vital role in the mechanisms underlying I/R-induced neuronal damage [4,5]. However, the relationship between autophagy and cerebral ischemia is far from clear.

Currently, there are two therapeutic strategies used in cerebral ischemia and reperfusion (I/R) injury. The most common is to restore blood flow by administering thrombolytic agents [6]. However, thrombolytic therapy can lead to hemorrhagic complications, and the strict 3 -hour time therapeutic window is the main barrier. Neuroprotection, as another strategy for treatment of cerebral ischemia and reperfusion injury, has attracted much attention in recent years [7].

Accumulating evidence has shown that phytoestrogens exert beneficial effects in a variety of neurodegenerative disorders, including acute ischemic stroke [8-10] Due to a structural similarity to the mammalian estrogen, phytoestrogens can selectively bind estrogen receptors (ERs) to regulate relevant gene expression and produce estrogenic and/or anti-estrogenic effects. Calycosin, as an isoflavone phytoestrogen isolated from Astragalus membranaceus, is found to possess various potential pharmacological activities. In previous studies, calycosin exerted anti-tumorigenesis, anti-oxidation, antivirus and apoptosis-modulation effects [11-14]. Additionally, our preliminary data have shown that calycosin has neuroprotective effects in cerebral I/R in rats via downregulation of RASD1 (Dexamethasone-induced Rasrelated protein 1) and upregulation of miR-375 [15]. However, the further neuroprotective mechanisms of calycosin involved in autophagy and apoptosis needed to be investigated. Therefore, the current study aimed to discuss the molecular mechanisms of calycosin effects against cerebral I/R injury in rats.

\section{Materials and Methods}

Drugs

Calycosin (purity $\geq 98.0 \%$, obtained from Phytomarker Ltd, Tianjin, China) (Fig. 1A) was dissolved in dimethyl sulfoxide (DMSO) to make a $200 \mathrm{mM}$ stock solution and stored at $4{ }^{\circ} \mathrm{C}$ for further use.

\section{Animal experiments}

Adult male Sprague-Dawley rats (220-250 g) were purchased from the Experimental Animal Center of Guilin Medical University. All rats were housed in a temperature-controlled $\left(22-23^{\circ} \mathrm{C}\right)$ environment under a 12-h light/ 12-dark cycle with free access to food and water. Experiments were conducted in accordance with animal care guidelines approved by the Animal Ethics Committee of the Guilin Medical University. All animal studies were performed according to the National Institute of Health Guide for the Care and Use of Laboratory Animals.

A model of focal cerebral ischemia and reperfusion used in this study was established as previously reported with some modifications [16]. Briefly, rats were anesthetized with $10 \%$ chloral hydrate (350 mg/ $\mathrm{kg}$, ip) $1 \mathrm{~h}$ after the last drug administration. The right common carotid artery and external carotid artery were exposed. A nylon filament with a silicon coated tip was inserted into the external carotid artery and advanced into the internal carotid artery until it blocked the origin of the middle cerebral artery (MCA), at which mild resistance was felt. During the surgery, the rectal temperature was maintained at $37-38{ }^{\circ} \mathrm{C}$ with a heating pad. After ischemia for $2 \mathrm{~h}$, the filament was gently removed, and animals were allowed to recover. For the sham-operated group, rats underwent the same surgical procedures, but without occlusion of the external carotid artery.

In this study, the experimental rats were randomly assigned to different groups $(n=10)$ : sham-operated group; I/R group; and calycosin-treated groups with different doses (intraperitoneal injection with calycosin $5,10,20 \mathrm{mg} / \mathrm{kg} / \mathrm{d}$ for 14 days).

\section{KARGER}




\section{Cellular Physiology Cell Physiol Biochem 2018;45:537-546

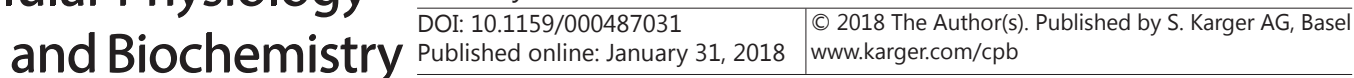

Wang et al.: Neuroprotective Mechanisms of Calycosin

\section{Neurological deficit scoring and infarct volume measurement}

After $2 \mathrm{~h}$ ischemia followed by $24 \mathrm{~h}$ reperfusion, the neurological deficit was evaluated according to the 5-point scoring system described by Longa et al [16].. Briefly, rats with a normal walk or no neurologic deficits were scored as 0 , with a failure to extend the opposite forepaw fully or with a mild focal neurologic deficit scored as 1, with circling to the contralateral side or with a moderate focal neurologic deficit scored as 2 , with falling to the contralateral side or with a severe focal neurologic deficit scored as 3 , and with no spontaneous walking and a depressed consciousness level scored as 4 . Neurological deficit scoring was performed by an investigator who was blinded to animal treatments.

After $24 \mathrm{~h}$ of reperfusion, the brains were immediately removed and sliced into coronal sections at $2 \mathrm{~mm}$ intervals with brain-cutting matrix. The slices were incubated in 2\% 2, 3,5-triphenyltetrazolium chloride (TTC) solution for $30 \mathrm{~min}$ at $37{ }^{\circ} \mathrm{C}$. The stained brain slices were photographed with a digital camera. Relative lesion volumes were calculated as the ratio of non TTC stained areas (white, infarct area) to total area, and expressed as a percentage. Image analysis software (NIH Image, National Institutes of Health, Bethesda, Version 1.63) was used to for measurement of the infracted area.

\section{Measurement of the brain edema}

The extent of cerebral edema was evaluated by measuring brain water content according to the wetdry method. In brief, brains were quickly removed and weighed to obtain the wet weight using an electronic analytic balance. Then, the samples were dried in an oven at $100^{\circ} \mathrm{C}$ for $24 \mathrm{~h}$ and weighed again to obtain the dry weight. The degree of brain water content was calculated as follows: water content = (wet weight - dry weight) / wet weight $\times 100 \%$.

\section{Immunohistochemical analyses}

At $24 \mathrm{~h}$ after reperfusion, rats were deeply anesthetized and perfused transcardially with normal saline, followed by 4\% PFA (paraformaldehyde) in PBS (phosphate buffered solution). The brains were harvested and fixed in 4\% PFA for 24h. A standard paraffin block was prepared, and a series of $5 \mu \mathrm{m}$ thick sections were cut. After being dewaxed and rehydrated, sections were incubated with primary antibodies (1:500; Abcam, Cambridge, MA, USA) at $37^{\circ} \mathrm{C}$ overnight. Sections were rinsed three times with PBS, and the sections were incubated with corresponding secondary antibodies (1:500; ZSGB-BIO, Beijing, China) for $1 \mathrm{~h}$ at 37 ${ }^{\circ} \mathrm{C}$ in a humidified chamber in the dark. Samples were subsequently stained with DAB (diaminobenzidine) and hematoxylin. The sections were digitized and analyzed under an optical microscope. Brown particles observed via optical microscopy in the cytoplasm or nucleus indicated positively stained cells. Five fields of view were randomly selected at 200× magnification for analyzing the integral optical density (IOD) value, using Image-Pro Plus 6.0.

\section{Quantitative real RT-PCR}

Total RNA was extracted from the ischemic penumbras of rat brains with Trizol reagent (Invitrogen, New York, USA), and reverse-transcription reactions were performed using the First Strand cDNA Synthesis Kit (TIANGEN, Beijing, China) according to the manufacturer's instructions. Target gene expressions (rat $B c l-2, N B R 1, p 62, T N F-\alpha$ ) were measured by quantitative real-time PCR using the ABI PRISM 7500 Sequence Detector System (Applied Biosystems, Carlsbad, CA, USA). The $2^{-\Delta \Delta C T}$ method was used to calculate the relative levels of gene expression. Transcriptional levels were normalized to $\beta$-actin in all samples.

\section{Western blotting test}

Rat brain tissues of the ischemic penumbras were separated and homogenized with RIPA lysis buffer (Beyotime, Nanjing, Jiangsu, China). The lysates were centrifuged at 12, $000 \mathrm{rpm}$ for $10 \mathrm{~min}$ at $4^{\circ} \mathrm{C}$. The supernatant protein concentration was measured with a BCA (bicinchoninic acid) protein assay kit (Beyotime, Nanjing, Jiangsu, China). Then, sodium dodecyl sulfate (SDS) sample loading buffer was added to the supernatant, and the mixture was boiled at $100{ }^{\circ} \mathrm{C}$ for $5 \mathrm{~min}$. Samples containing $40 \mu \mathrm{g}$ protein per lane were separated using SDS-PAGE (sodium dodecyl sulfate polyacrylamide gel electrophoresis) and transferred to PVDF (polyvinylidene fluoride) membranes (Millipore, Bedford, MA, USA). Blots were blocked with TBST (tris buffered saline with Tween 20) containing 5\% nonfat dried milk for $1 \mathrm{~h}$. Following blocking, the membranes were incubated overnight at $4{ }^{\circ} \mathrm{C}$ with the following primary antibodies: Bcl-2, NBR1 (neighbor of BRCA1 gene 1), p62, TNF- $\alpha$ (Santa Cruz, Dallas, Texas, USA; 1:1000), and mouse anti-rat 
Wang et al.: Neuroprotective Mechanisms of Calycosin

$\beta$-actin antibody (1:500; ZSGB-BIO, Beijing, China; $1: 2000)$. After three washes in TBST, the membranes were subsequently incubated with the appropriate secondary antibodies coupled to horseradish peroxidase at room temperature for $1 \mathrm{~h}$. Protein bands was scanned and visualized using an enhanced chemiluminescence detection system (Life Technologies, Pleasanton, CA, USA). The optical density of the bands was quantified with a computer-assisted image analysis system (Bio-Rad Laboratories, Hercules, CA, USA) and normalized to $\beta$-actin protein level.

\section{Statistical analysis}

All data were expressed as the mean \pm Standard Error (S.E.). The Statistical Package for Social Sciences (SPSS) 13.0 software (SPSS, Chicago, IL) was used for statistical analyses, including a one-way ANOVA and Student's t-test. A probability (p)-value $<0.05$ was considered statistically significant.

\section{Results}

Treatment of calycosin attenuated I/R-induced neurological impairments and decreased the ischemic infarct area and brain edema

To determine whether calycosin treatment led to an improvement in neurological function after ischemia, neurological deficit scoring was carried out. As shown in Fig. 1B, rats in the sham-operated group presented no neurologic deficits, while obvious neurological deficits were observed in the I/R group, suggesting that the MCAO model was successfully constructed. Additionally, 5-20 mg/kg of calycosin reduced the neurological deficit scores in a dose-dependent manner, indicating that calycosin ameliorated the brain dysfunction.

In rats subjected to MCA occlusion for $2 \mathrm{~h}$ followed by $24 \mathrm{~h}$ reperfusion, an increase in the infarct area and brain edema was also observed. As shown in Fig. 1C and 1D, the infarct size and brain water content in the I/R group obviously increased compared to that in the shamoperated group, while calycosin significantly decreased the infarct size and brain water content dosedependently. These results suggest that calycosin alleviated the damage in the ischemic brain areas.

Calycosin increased p62 and NBR1 expression in cerebral ischemia and reperfusion rats

It has been demonstrated that p62 and NBR1 are substrates for selective

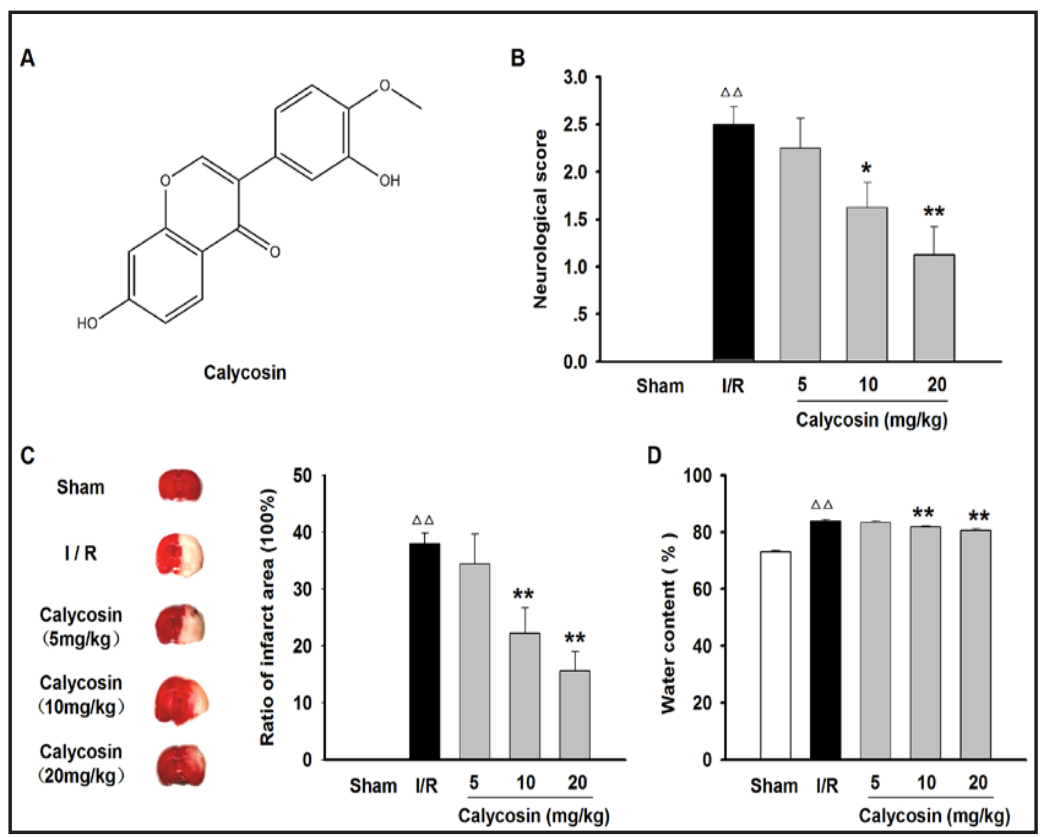

Fig. 1. The neuroprotective effects of calycosin on cerebral I/R in rats. (A) The basic structure of calycosin. (B) A 5-point scoring system was used to evaluate the neurological deficits at $24 \mathrm{~h}$ after reperfusion. The brains were collected and stained with TTC for $30 \mathrm{~min}$ at $37^{\circ} \mathrm{C}$. (C) Representative samples of TTC-stained brain slices and statistical analysis of the percentage of infarct area after $24 \mathrm{~h}$ reperfusion following $2 \mathrm{~h}$ ischemia. (D) The percentage of brain edema in each group was measured by the wet-dry method. The results are expressed as the mean \pm SE. ${ }^{\Delta} \mathrm{P}<0.01$, I/R vs the Sham group; ${ }^{*} \mathrm{P}<0.05,{ }^{* *} \mathrm{P}<0.01$, vs the I/R group, $n=8$. 
Wang et al.: Neuroprotective Mechanisms of Calycosin

Fig. 2. Effects of calycosin on expression levels of p62 in cerebral ischemia and reperfusion rats. Tissue from the penumbra was collected at $24 \mathrm{~h}$ after reperfusion and used for Quantitative real RT-PCR and western blot analysis. Sections obtained from formalin-fixed, paraffin-embedded brain tissue specimens were used for immunohistochemical analyses. (A) Calycosin increased p62 mRNA expression levels in I/R rats. (B) Top panel and bottom panel are representative bands and $\mathrm{p} 62$ relative protein levels. Calycosin increased p62 protein expression levels in $I / R$

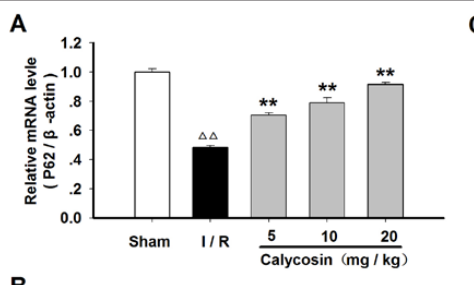

B

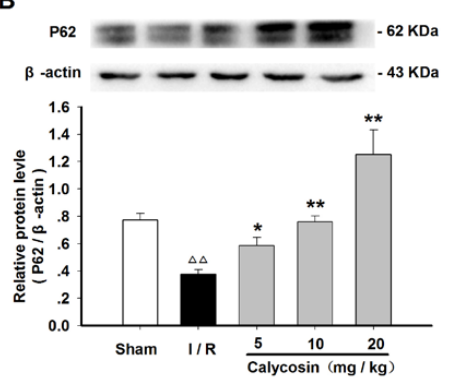

C
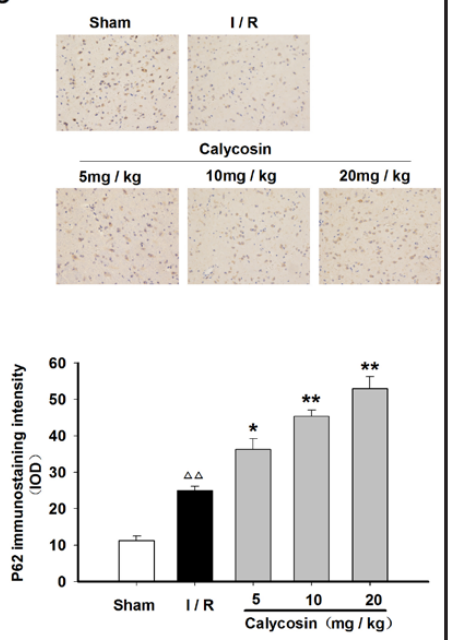
rats. (C) Representative p62 immu-

nohistochemically stained sections and semiquantitative density results. Calycosin treatment significantly increased the immunohistochemical staining expression of p62 compared with the I/R group. The results are expressed as the mean $\pm \mathrm{SE} .{ }^{\Delta} \mathrm{P}<0.01$, I/R vs the Sham group; ${ }^{*} \mathrm{P}<0.05,{ }^{* *} \mathrm{P}<0.01$, vs the I/R group, $\mathrm{n}=3$.

Fig. 3. Effects of calycosin on expression levels of NBR1 in cerebral ischemia and reperfusion rats. Tissue from the penumbra was collected at $24 \mathrm{~h}$ after reperfusion and used for Quantitative real RTPCR and western blot analysis. Sections obtained from formalinfixed, paraffin-embedded brain tissue specimens were used for immunohistochemical analyses. (A) Calycosin increased NBR1 mRNA expression levels in $I / R$ rats. (B) Top panel and bottom panel are representative bands and NBR1 relative protein levels. Calycosin increased NBR1 protein

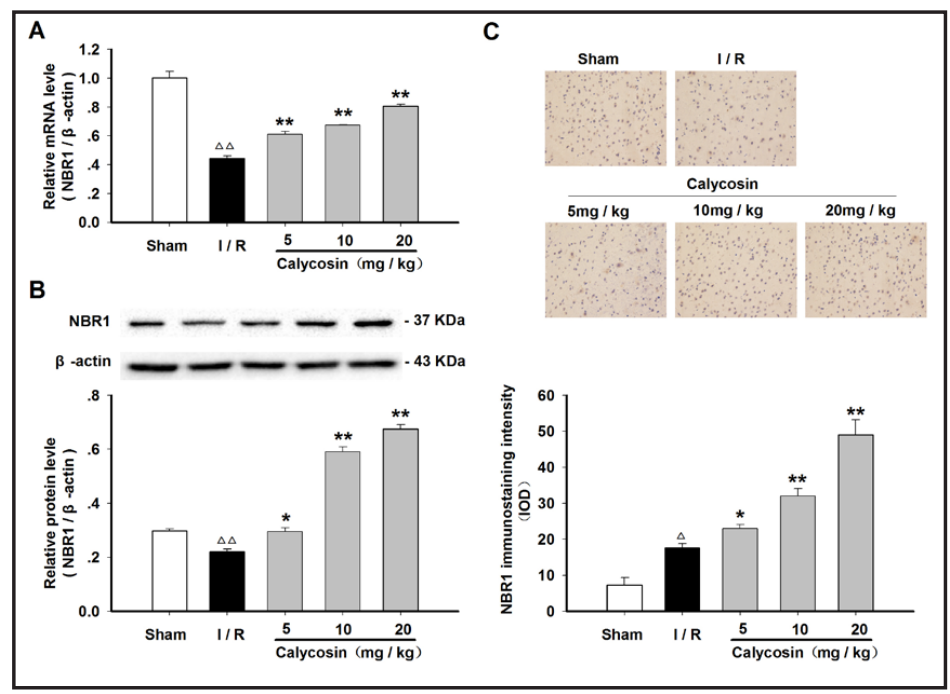
expression levels in $\mathrm{I} / \mathrm{R}$ rats.

(C) Representative NBR1 immunohistochemically stained sections and semiquantitative density results. Calycosin treatment significantly increased the immunohistochemical staining expression of NBR1 compared with the I/R group. The results are expressed as the mean $\pm S E .{ }^{\Delta} \mathrm{P}<0.01, \mathrm{I} / \mathrm{R}$ vs the Sham group; ${ }^{*} \mathrm{P}<0.05,{ }^{*} \mathrm{P}<0.01$, vs the I/R group, $\mathrm{n}=3$.

autophagy. The mRNA and protein levels of the above two molecules related to autophagy were examined in I/R injury brain tissue. As Fig. 2A-B and Fig. 3A-B illustrate, the relative expression of both p62 and NBR1 was significantly downregulated in the I/R group, and calycosin significantly increased the expression of the two molecules in a dose-dependent manner. Meanwhile, immunohistochemical analyses showed that the number of p62 and NBR1 positive cells in calycosin-treated I/R rat brains were also increased in comparison to those in the I/R group (Fig. 2C and Fig. 3C). The results indicate that calycosin may achieve a neuroprotective effect via inhibition of the autophagy activated by I/R injury.

\section{KARGER}


Wang et al.: Neuroprotective Mechanisms of Calycosin

Fig. 4. Effects of calycosin on expression levels of Bcl-2 in cerebral ischemia and reperfusion rats. Tissue from the penumbra was collected at $24 \mathrm{~h}$ after reperfusion and used for Quantitative real RT-PCR and western blot analysis. Sections obtained from formalinfixed, paraffin-embedded brain tissue specimens were used for immunohistochemical analyses. (A) Calycosin increased Bcl-2 mRNA expression levels in $\mathrm{I} / \mathrm{R}$ rats. (B) Top panel and bottom panel are representative bands and $\mathrm{Bcl}-2$ relative protein levels. Calycosin increased Bcl-2 protein expression

A

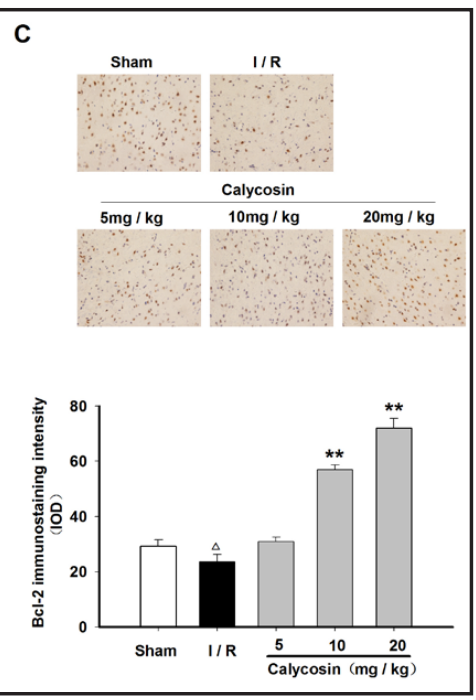
levels in I/R rats. (C) Representative Bcl-2 immunohistochemically stained sections and semiquantitative density results. Calycosin treatment significantly increased the immunohistochemical staining expression of Bcl-2 compared with the I/R group. The results are expressed as the mean $\pm \mathrm{SE} .{ }^{\Delta} \mathrm{P}<0.01$, I/R vs the Sham group; ${ }^{*} \mathrm{P}<0.05,{ }^{* *} \mathrm{P}<0.01$, vs the I/R group, $\mathrm{n}=3$.

Fig. 5. Effects of calycosin on expression levels of TNF- $\alpha$ in cerebral ischemia and reperfusion rats. Tissue from the penumbra was collected at $24 \mathrm{~h}$ after reperfusion and used for Quantitative real RTPCR and western blot analysis. Sections obtained from formalinfixed, paraffin-embedded brain tissue specimens were used for immunohistochemical analyses. (A) Calycosin decreased TNF- $\alpha$ mRNA expression levels in $I / R$ rats. (B) Top panel and bottom panel are representative bands and TNF- $\alpha$ relative protein levels. Calycosin decreased TNF- $\alpha$ protein expression levels in $\mathrm{I} / \mathrm{R}$

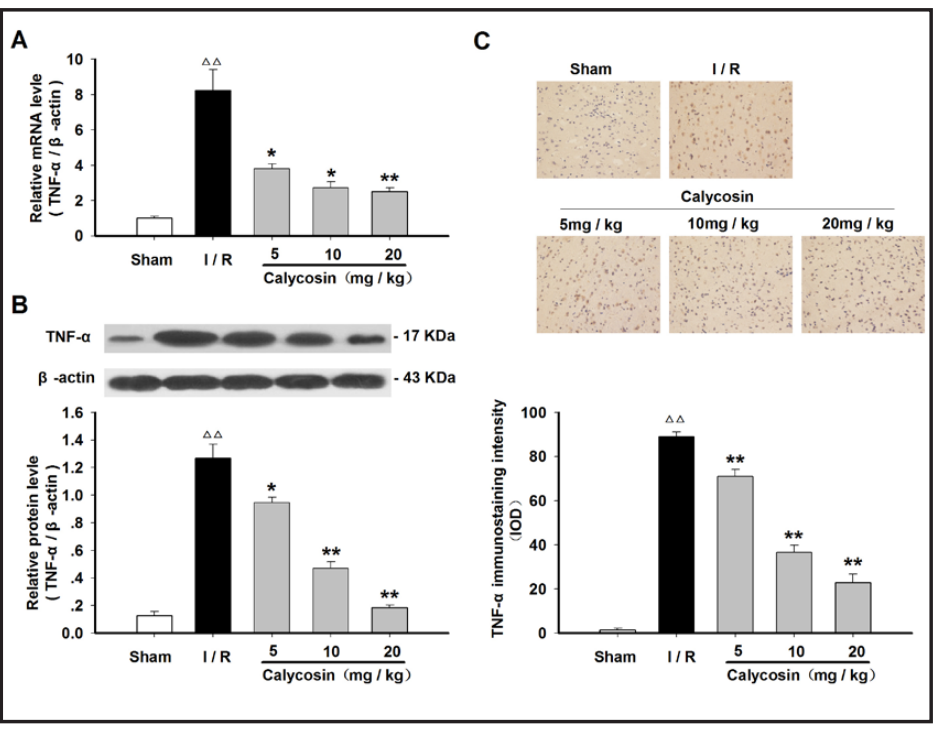
rats. (C) Representative TNF- $\alpha$

immunohistochemically stained sections and semiquantitative density results. Calycosin treatment significantly decreased the immunohistochemical staining expression of TNF- $\alpha$ compared with the I/R group. The results are expressed as the mean \pm SE. ${ }^{\Delta} \mathrm{P}<0.01$, I/R vs the Sham group; ${ }^{*} \mathrm{P}<0.05,{ }^{* *} \mathrm{P}<0.01$, vs the I/R group, $\mathrm{n}=3$.

\section{Calycosin increased Bcl-2 expression in cerebral ischemia and reperfusion rats}

To investigate calycosin-triggered anti-apoptosis, we examined mRNA and proteins expression of Bcl-2. As shown in Fig. 4A-B, RT-PCR and western blot results suggested that less Bcl-2 mRNA and protein were observed in the I/R group compared with those in the shamoperated group $(P<0.05)$, while mRNA and protein levels of $B c l-2$ were gradually increased 


\section{Cellular Physiology Cell Physiol Biochem 2018;45:537-546 \\ \begin{tabular}{ll|l} 
DOI: 10.1159/000487031 & $\begin{array}{l}\text { O 2018 The Author(s). Published by S. Karger AG, Basel } \\
\text { www.karger.com/cpb }\end{array}$
\end{tabular}}

Wang et al.: Neuroprotective Mechanisms of Calycosin

following calycosin treatment. Additionally, as revealed in the immunohistochemistry observations, Bcl-2 positive cells in the calycosin-treated groups also increased compared with those in the I/R group (Fig. 4C). Based on the above results, calycosin exhibited antiapoptotic effects during the process of I/R brain injury.

\section{Calycosin inhibited TNF- $\alpha$ expression in cerebral ischemia and reperfusion rats}

To further analyze calycosin-induced anti-inflammatory effects, we examined mRNA and protein expression levels of TNF- $\alpha$. As shown in Fig. 5A-B, levels of TNF- $\alpha$ were significantly elevated in the I/R group compared with those in the sham-operated group $(P<0.01)$. Treatments with calycosin markedly attenuated the increase of TNF- $\alpha$ in a dose-dependent manner $(P<0.01$ or $P<0.05)$. In accordance with the mRNA and protein expression results, immunohistochemistry analyses also showed that TNF- $\alpha$ positive cells gradually reduced in number following calycosin treatment (Fig. 5C). These results indicate that calycosin may produce neuroprotection by suppressing neuroinflammation in cerebral I/R rats.

\section{Discussion}

It is generally accepted that neuroprotective therapy is an effective strategy to reduce neuronal damage during the process of cerebral I/R. However, experimental searches for neuroprotective agents and their mechanisms have so far failed to reveal effective agents. Accumulating evidence indicates that estrogens and estrogen-like compounds are powerful agents to protect neurons from neuropathological and neurotoxic challenges and promote their survival [17-19]. In the present study, calycosin, a typical isoflavone phytoestrogen, markedly improved neurological deficits and reduced both infarct size and brain edema. Moreover, administration of calycosin increased the expression of p62, NBR1 and Bcl-2, and reduced the expression of TNF- $\alpha$. Collectively, these data suggest that calycosin provides neuroprotection for cerebral I/R rats via key molecules involved in autophagy, apoptosis and inflammation.

Previous studies have identified at least three pathways of cell death in ischemic brain injury, including necrosis, apoptosis and autophagy [20]. Cerebral I/R injury could initiate physiopathologic cascades of cell death signaling and induce multiple cell death modes. In recent years, scholars have confirmed that autophagy can be induced in brain tissues or neuronal cells after ischemic stimulation [4, 21]. Autophagy is a conserved degradation pathway that delivers cytoplasmic proteins or damaged organelles to lysosome during stress conditions.

P62 (also known as sequestosome-1/ SQSTM1) is a selective substrate of autophagy and a multidomain scaffold protein [22]. Due to the presence of the LC3-interacting region (LIR) and a C-terminal ubiquitin associated (UBA) domain, p62 can interact with LC3 (a mammalian homologue of Atg8) and bind to mono- or poly-ubiquitin. Therefore, p62 is considered to act as a receptor for ubiquitinated cargos, including ubiquitin-tagged proteins and whole organelles to be selectively degraded. Similar to p62, NBR1 also contains a LIR and a C-terminal UBA domain and can mediate the degradation of ubiquitinated cargos through their interaction with ubiquitin [23]. Since p62 and NBR1 are helpful in scaffolding misfolded or mutated proteins to form aggregates, and ultimately autophagic degradation, they may be used as markers to study autophagic flux. Recent data proposed that decreased levels of p62 can be observed when autophagy is induced and that p62 accumulates when autophagy is inhibited [24, 25]. Angeles Duran et al. found that p62 can suppress autophagy via activation of mTORC1 [26]. In addition, p62 is an important adaptor protein for several signaling pathways that regulate apoptosis, stress responses, inflammatory processes, and cell growth by interaction with a large number of proteins, including caspase-8, Keap1, Nrf2, TRAF6, NF- $\kappa B$, etc. $[27,28]$. Our results showed that calycosin leads to activation of p62 and NBR1, suggesting that calycosin inhibits autophagy and thus contributes its cerebral protective effect. The possible anti-autophagy mechanisms of calycosin may be related to 
Wang et al.: Neuroprotective Mechanisms of Calycosin

increasing the levels of p62 and NBR1, and thereby regulating misfolded or mutated proteins to ubiquitination and degradation during I/R injury.

Apoptosis has been implicated in cerebral ischemic injury. Bcl-2, as a principal member of the Bcl-2 family that regulates apoptosis, is specifically regarded as an anti-apoptotic protein. Bcl-2 plays an important role in promoting cellular survival and inhibiting the actions of proapoptotic proteins. Recent studies showed that there exists an intricate crosstalk between apoptosis and autophagy [29]. According to a previous study by Levine et al., Bcl-2 inhibits Beclin-1-dependent autophagy, thereby functioning both as pro-survival and anti-autophagy factors [30]. The present results demonstrate that the neuroprotective effects of calycosin might associate with an attenuation of ischemia-induced apoptosis through upregulation of the level of Bcl-2.

Inflammatory responses are considered to be the key element in the pathological progression of cerebral ischemia and reperfusion [31]. Following ischemic stress, damaged brain tissue secretes cytokines and chemokines that recruit inflammatory cells to the injured area. As one of the early initiators of inflammation after stroke, TNF $\alpha$ mediates apoptosis signals via the FADD (Fas associated death domain) of TNF-R1, and inflammatory responses via NF- $\chi \mathrm{B}$ [32]. Apart from apoptosis and autophagy, $\mathrm{p} 62$ is also involved in the regulation of NF- $\mathrm{KB}$ signaling pathways. Wooten MW et al. reported that p62 is needed for NF- $\chi \mathrm{B}-$ mediated neuronal survival and differentiation in response to NGF (nerve growth factor) [33]. Our results showed that the inhibitory effect of calycosin on the pro-inflammatory cytokine TNF $\alpha$ may be one of the crucial mechanisms in its effects against cerebral I/R injury. Additionally, reducing the levels of inflammatory mediators such as TNF $\alpha$ is the probable basis for ameliorating brain edema with treatment of calycosin.

In general, these findings suggest that cerebral I/R injury coincides with pathology associated changes in autophagy, apoptosis and inflammatory responses. The influence of the phytoestrogen calycosin on cerebral I/R injury is of practical importance for a variety of neurologic dysfunctions, in particular ischemic stroke. However, a limitation of this study should be noted: we did not determine the specific signaling pathways of calycosin in autophagy, apoptosis and inflammation regulation. A greater understanding of the neuroprotective molecular mechanisms of calycosin is needed by further study.

\section{Conclusion}

Taken together with our findings, the phytoestrogen calycosin significantly protected against cerebral ischemia and reperfusion injury in rats. The mechanism by which calycosin exerts its neuroprotective activity, at least partly, is its association with inhibiting the processes of autophagy, apoptosis and inflammation in the I/R-injured brain. These results suggest that calycosin can be further developed as a clinical neuroprotective candidate for treatment of ischemic stroke.

\section{Acknowledgements}

This study was supported by agrant from the National Natural Science Foundation of China (NSFC, No. 81460554), the Natural Science Foundation of Guangxi (2015GXNSFBB139006), and the Scientific Research Project of Guangxi Higher Education Institutions (No. KY2015ZD090).

\section{Disclosure Statement}

The authors declare that there are no conflicts of interest. 


\section{Cellular Physiology Cell Physiol Biochem 2018;45:537-546 and Biochemistry Published online: January 31, 2018 \begin{tabular}{l|l} 
DOI: 101159/2 The Author(s). Published by S. Karger AG, Basel \\
www.karger.com/cpb
\end{tabular}}

Wang et al.: Neuroprotective Mechanisms of Calycosin

\section{References}

1 Khoshnam SE, Winlow W, Farzaneh M, Farbood Y, Moghaddam HF: Pathogenic mechanisms following ischemic stroke. Neurol Sci 2017;38:1167-1186.

2 Chomova M, Zitnanova I: Look into brain energy crisis and membrane pathophysiology in ischemia and reperfusion. Stress 2016;19:341-348.

-3 Durukan A, Tatlisumak T: Acute ischemic stroke: overview of major experimental rodent models, pathophysiology, and therapy of focal cerebral ischemia. Pharmacol Biochem Behav 2007;87:179-197.

4 Wen YD, Sheng R, Zhang LS, Han R, Zhang X, Zhang XD, Han F, Fukunaga K, Qin ZH: Neuronal injury in rat model of permanent focal cerebral ischemia is associated with activation of autophagic and lysosomal pathways. Autophagy 2008;4:762-769.

5 Puyal J, Ginet V, Clarke PG: Multiple interacting cell death mechanisms in the mediation of excitotoxicity and ischemic brain damage: a challenge for neuroprotection. Prog Neurobiol 2013;105:24-48.

6 Saver JL: Improving reperfusion therapy for acute ischaemic stroke. J Thromb Haemost 2011;9:S333-343.

7 Patel RAG, McMullen PW: Neuroprotection in the Treatment of Acute Ischemic Stroke. Prog Cardiovasc Dis 2017;59:542-548.

8 Menze ET, Esmat A, Tadros MG, Abdel-Naim AB, Khalifa AE: Genistein improves 3-NPA-induced memory impairment in ovariectomized rats: impact of its antioxidant, anti-inflammatory and acetylcholinesterase modulatory properties. PLoS One 2015;10:e0117223.

-9 Schreihofer DA, Redmond L: Soy phytoestrogens are neuroprotective against stroke-like injury in vitro. Neuroscience 2009;158:602-609.

$>10$ Subedi L, Ji E, Shin D, Jin J, Yeo JH, Kim SY: Equol, a Dietary Daidzein Gut Metabolite Attenuates Microglial Activation and Potentiates Neuroprotection In vitro. Nutrients 2017;9:207

11 Chen J, Lin C, Yong W, Ye Y, Huang Z: Calycosin and genistein induce apoptosis by inactivation of HOTAIR/pAkt signaling pathway in human breast cancer MCF-7 cells. Cell Physiol Biochem 2015;35:722-728.

-12 Zhao X, Li X, Ren Q Tian J, Chen J: Calycosin induces apoptosis in colorectal cancer cells, through modulating the ERbeta/MiR-95 and IGF-1R, PI3K/Akt signaling pathways. Gene 2016;591:123-128.

$>13$ Wang X, Zhao L: Calycosin ameliorates diabetes-induced cognitive impairments in rats by reducing oxidative stress via the PI3K/Akt/GSK-3beta signaling pathway. Biochem Biophys Res Commun 2016;473:428-434.

14 Zhu H, Zhang Y, Ye G, Li Z, Zhou P, Huang C: In vivo and in vitro antiviral activities of calycosin-7-0-beta-Dglucopyranoside against coxsackie virus B3 Biol Pharm Bull 2009;32:68-73.

15 Wang Y, Dong X, Li Z, Wang W, Tian J, Chen J: Downregulated RASD1 and upregulated miR-375 are involved in protective effects of calycosin on cerebral ischemia/reperfusion rats. J Neurol Sci 2014;339:144-148.

16 Longa EZ, Weinstein PR, Carlson S, Cummins R: Reversible middle cerebral artery occlusion without craniectomy in rats. Stroke 1989;20:84-91.

17 Ma Y, Guo H, Zhang L, Tao L, Yin A, Liu Z, Li Y, Dong H, Xiong L, Hou W: Estrogen replacement therapyinduced neuroprotection against brain ischemia-reperfusion injury involves the activation of astrocytes via estrogen receptor beta. Sci Rep 2016;6:21467.

18 Engler-Chiurazzi EB, Brown CM, Povroznik JM, Simpkins JW: Estrogens as neuroprotectants: Estrogenic actions in the context of cognitive aging and brain injury. Prog Neurobiol 2017;157:188-211.

19 Simpkins JW, Yang SH, Liu R, Perez E, Cai ZY, Covey DF, Green PS: Estrogen-like compounds for ischemic neuroprotection. Stroke 2004;35:2648-2651.

20 Lipton P: Ischemic cell death in brain neurons. Physiol Rev 1999;79:1431-1568.

-21 Lu Q Harris VA, Kumar S, Mansour HM, Black SM: Autophagy in neonatal hypoxia ischemic brain is associated with oxidative stress. Redox Biol 2015;6:516-523.

-22 Johansen T, Lamark T: Selective autophagy mediated by autophagic adapter proteins. Autophagy 2011;7:279-296.

23 Deng Z, Purtell K, Lachance V, Wold MS, Chen S, Yue Z: Autophagy Receptors and Neurodegenerative Diseases. Trends Cell Biol 2017;27:491-504.

24 Puyal J, Vaslin A, Mottier V, Clarke PG: Postischemic treatment of neonatal cerebral ischemia should target autophagy. Ann Neurol 2009;66:378-389. 


\section{Cellular Physiology Cell Physiol Biochem 2018:45:537-546

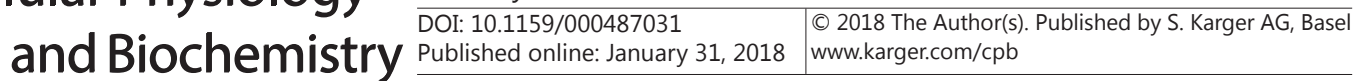

Wang et al.: Neuroprotective Mechanisms of Calycosin

25 Zhang XJ, Chen S, Huang KX, Le WD: Why should autophagic flux be assessed? Acta Pharmacol Sin 2013;34:595-599.

26 Duran A, Amanchy R, Linares JF, Joshi J, Abu-Baker S, Porollo A, Hansen M, Moscat J, Diaz-Meco MT: p62 is a key regulator of nutrient sensing in the mTORC1 pathway. Mol Cell 2011;44:134-146.

27 Moscat J, Diaz-Meco MT: p62 at the crossroads of autophagy, apoptosis, and cancer. Cell 2009;137:10011004.

-28 Komatsu M, Kageyama S, Ichimura Y: p62/SQSTM1/A170: physiology and pathology. Pharmacol Res 2012;66:457-462.

29 Thorburn A: Apoptosis and autophagy: regulatory connections between two supposedly different processes. Apoptosis 2008;13:1-9.

30 Levine B, Sinha S, Kroemer G: Bcl-2 family members: dual regulators of apoptosis and autophagy. Autophagy 2008;4:600-606.

-31 Esenwa CC, Elkind MS: Inflammatory risk factors, biomarkers and associated therapy in ischaemic stroke. Nat Rev Neurol 2016;12:594-604.

-32 Liu ZG: Molecular mechanism of TNF signaling and beyond. Cell Res 2005;15:24-27.

33 Wooten MW, Seibenhener ML, Mamidipudi V, Diaz-Meco MT, Barker PA, Moscat J: The atypical protein kinase C-interacting protein p62 is a scaffold for NF-kappaB activation by nerve growth factor. J Biol Chem 2001;276:7709-7712. 